\title{
Various Domination Parameters of Single Valued Neutrosophic Graphs
}

\author{
R. Jahir Hussain 1 and S. Satham Hussain \\ PG and Research Department of Mathematics, \\ Jamal Mohamed College, Trichy - 620 020, Tamil Nadu, India. E-mail: hssn_jhr@yahoo.com, \\ sathamhussain5592@gmail.com.
}

\begin{abstract}
In this manuscript, we present the concept of single valued neutrosophic graph structures. We introduce the domination number of single valued neutrosophic graphs and elaborate them with suitable examples by using strength of path and strength of connectedness. Further, we investigate some remarkable properties of independent domination number, product domination, triple connected domination of single valued neutrosophic graph and the proposed concepts are described with suitable examples.
\end{abstract}

Keywords: Domination Number, Single valued Neutrosophic graphs, Triple Connected domination, Product Domination.

\section{INTRODUCTION}

Fuzzy graph theory was introduced by Azriel Rosenfied in 1975. Though it is very young it has been growing very fast and has crucial applications in varies fields. Fuzzy set was introduced by Zadeh 29] whose basic components is only a membership function. The generalization of Zadeh's fuzzy set, called intuitionistic fuzzy set was introduced by atanassov which is characterized by a membership function and a non membership function. According to Atanassov, the sum of membership degree and a non membership degree does not exceed one. A. Somasundaram and S. Somasundaram [5] presented more concept of independent domination, connected domination in fuzzy graphs, R. Parvathi and G. Thamilzhendhi 3 introduced domination in intuitionistic fuzzy graphs and discussed some of its properties. Molodtsov 19 introduced the concept of soft set theory as a new mathematical tool for dealing with uncertainties. Molodtsov's soft sets give us new technique for dealing with uncertainty from the view point of parameters. It has been revealed that soft sets have potential applications in several fields. Recently, the author Smarandache [17, 18, 25, 29] introduced and studied extensively about neutrosophic set and it receives applications in many domains. Akram [7] established the certain notions including neutrosophic soft graphs, strong neutrosophic soft graphs, complete neutrosophic soft graphs. Motivation of the above, we introduced the concept of domination number in single valued neutrosophic fuzzy graphs, product domination and triple connected domination number in single valued neutrosophic fuzzy graphs. The major contribution of this work as follows:

- The domination number of single valued neutrosophic soft graphs is established by using the concept of strength of a path, strength of connectedness and strong arc.

- The necessary and sufficient condition for the minimum domination set of single valued neutrosophic soft graph is investigated.

- Some properties of independent domination number of single valued neutrosophic soft graph are obtained and the proposed concepts are described with suitable examples.

- Further we presented a remarkable properties of product domination of single valued neutrosophic soft graph.

\section{PRELIMINARIES}

Definition 2.1. A Neutrosophic set $A$ is contained in another neutrosophic set $B$, (i.e) $A \subseteq C$ if $\forall x \in X, T_{A}(x) \leq T_{B}(x), I_{A}(x) \leq I_{B}(x)$ and $F_{A}(x) \geq F_{B}(x)$.

Definition 2.2. Let $X$ be a space of points (objects), with a generic elements in $X$ denoted by $x$. $A$ single valued neutrosophic set (SVNS) $A$ in $X$ is characterized by truth-membership function $T_{A}(x)$,

${ }^{1}$ Corresponding Author 
indeterminacy-membership function $I_{A}(x)$ and falsity-membership-function $F_{A}(x)$. For each point $x$ in $X, T_{A}(x), F_{A}(x), I_{A}(x) \in[0,1]$.

$$
A=\left\{x, T_{A}(x), F_{A}(x), I_{A}(x)\right\} \text { and } 0 \leq T_{A}(x)+I_{A}(x)+F_{A}(x) \leq 3
$$

Definition 2.3. A single-valued neutrosophic graph (SVNG) is defined as a pair $G=(V, E)$ where $(i)$ $V=\left\{v_{1}, v_{2}, . ., v_{n}\right\}$ such that $T_{1}=V \rightarrow[0,1], I_{1}=V \rightarrow[0,1]$ and $F_{1}=V \rightarrow[0,1]$ denote the degree of truth-membership function, indeterminacy function and falsity-membership function, respectively and

$$
0 \leq T_{A}(x)+I_{A}(x)+F_{A}(x) \leq 3 .
$$

(ii) $E \subset V \times V$ where $T_{2}=E \rightarrow[0,1], I_{2}=E \rightarrow[0,1]$ and $F_{2}=E \rightarrow[0,1]$ are such that

$$
\begin{aligned}
T_{2}(u v) & \leq \min \left\{T_{1}(u), T_{1}(v)\right\}, \\
I_{2}(u v) & \leq \min \left\{I_{1}(u), I_{1}(v)\right\}, \\
F_{2}(u v) & \leq \max \left\{F_{1}(u), F_{1}(v)\right\} .
\end{aligned}
$$$$
\text { and } 0 \leq T_{2}(u v)+I_{2}(u v)+F_{2}(u v) \leq 3 . \forall u v \in E
$$

Definition 2.4. The order of a $(S V N G) G$ is $\operatorname{Ord}(G)=\sum_{x \in V} T_{J}(x), \sum_{x \in V} I_{J}(x), \sum_{x \in V} F_{J}(x)$

Definition 2.5. The size of a $(S V N G) G$ is $\operatorname{Size}(G)=\sum_{x \in E} T_{K}(x y), \sum_{x \in E} I_{K}(x y), \sum_{x \in E} F_{K}(x y)$

Definition 2.6. Let $G=(V, E)$ be an $(S V N G)$. then cardinality of $G$ is defined to be $|G|=\left|\sum_{v_{i \in V}} \frac{1+T_{J(e)}(x)+I_{\left.J_{(} e\right)}(x)-F_{J(e)}(x)}{2}+\sum_{v_{i v_{j} \in E}} \frac{1+T_{K(e)}(x y)+I_{K_{(}(e)}(x y)-F_{K(e)}(x y)}{2}\right| \forall u v \in V$

Definition 2.7. Let $G=(V, E)$ be an $(S V N G)$, then vertex cardinality of $G$ is defined to be

$$
|V|=\left|\sum_{x \in V} \frac{1+T_{J(e)}(x)+I_{\left.J_{(}\right)}(x)-F_{J(e)}(x)}{2}\right| \forall u v \in V .
$$

Definition 2.8. Let $G=(V, E)$ be an $(S V N G)$, then the Edge cardinality of $E$ is defined to be $|E|=\left|\sum_{x y \in E} \frac{1+T_{K(e)}(x y)+I_{K(e)}(x y)-F_{K(e)}(x y)}{2}\right| \forall u v \in E$.

Definition 2.9. The degree of a vertex $v$ in an (SVNG) $G$ is defined to be sum of the weight of the strong edges incident at $v$. It is denoted by $d_{G}(v)$.

The minimum degree of $G$ is $\delta(G)=\min \left\{d_{g}(v) / v \in V.\right\}$

The maximum degree of $G$ is $\Delta(G)=\max \left\{d_{g}(v) / v \in V\right.$. $\}$

Definition 2.10. Two vertices $x$ and $y$ are said to be neighbors in (SVNG) graph if either one of the following conditions hold.

(1) $T_{K(e)}(x y)>0, I_{K(e)}(x y)>0, F_{K(e)}(x y)>0$,

(2) $T_{K(e)}(x y)>0, I_{K(e)}(x y)=0, F_{K(e)}(x y)>0$,

(3) $T_{K(e)}(x y)>0, I_{K(e)}(x y)>0, F_{K(e)}(x y)=0$,

(4) $T_{K(e)}(x y)=0, I_{K(e)}(x y)>0, F_{K(e)}(x y)>0$. for all $x, y \in V$.

Definition 2.11. A path in an $(S V N G)$ is a sequence of distinct vertices $v_{1}, v_{2}, \ldots, v_{n}$. such that either one of the following conditions is satisfied.

(1) $T_{K(e)}(x y)>0, I_{K(e)}(x y)>0, F_{K(e)}(x y)>0$,

(2) $T_{K(e)}(x y)>0, I_{K(e)}(x y)=0, F_{K(e)}(x y)>0$,

(3) $T_{K(e)}(x y)>0, I_{K(e)}(x y)>0, F_{K(e)}(x y)=0$,

(4) $T_{K(e)}(x y)=0, I_{K(e)}(x y)>0, F_{K(e)}(x y)>0$. for all $x, y \in V$,

Definition 2.12. The length of a path ( $S V N G) P=v_{1}, v_{2}, \ldots, v_{n+1}(n>0)$ is $n$.

Definition 2.13. If $v_{i}, v_{j}$ are vertices in $G$ and if they are connected means of a path then the strength of that path is defined as

$\left(\min _{i, j} T_{K(e)}\left(v_{i}, v_{j}\right), \min _{i, j} I_{K(e)}\left(v_{i}, v_{j}\right), \max _{i, j} F_{K(e)}\left(v_{i}, v_{j}\right)\right)$ where 
$\min _{i, j} T_{K(e)}\left(v_{i}, v_{j}\right)$ is the $T_{K(e)^{-}}$strength of weakest arc and $\min _{i, j} I_{K(e)}\left(v_{i}, v_{j}\right)$ is the $I_{K(e)^{-}}$strength of weakest arc and $\left.\max _{i, j} F_{K(e)}\left(v_{i}, v_{j}\right)\right)$ is the $F_{K(e)}$ - strength of strongest arc.

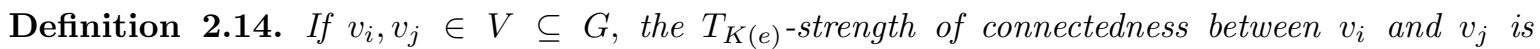
$T_{K(e)}^{\infty}\left(v_{i}, v_{j}\right)=\sup \left\{T_{K(e)}^{K}\left(v_{i}, v_{j}\right) / k=1,2, \ldots n, e \in A\right\}$ and $I_{K(e)}-$ strength of connectedness between $v_{i}$ and $v_{j}$ is $I_{K(e)}^{\infty}\left(v_{i}, v_{j}\right)=\sup \left\{I_{K(e)}^{k}\left(v_{i}, v_{j}\right) / k=1,2, \ldots n, e \in A\right\}$ and

$F_{K(e)}^{\infty}\left(v_{i}, v_{j}\right)=\inf \left\{F_{K(e)}^{k}\left(v_{i}, v_{j}\right) / k=1,2, \ldots n.\right\}$.

If $u, v$ are connected by means of path of length $k$ then $T_{K(e)}^{k}\left(v_{i}, v_{j}\right)$ is defined as $\sup \left\{T_{K(e)}\left(u, v_{1}\right) \wedge T_{K(e)}\left(v_{1}, v_{2}\right) \wedge T_{K(e)}\left(v_{2}, v_{3}\right) \ldots, T_{K(e)}\left(v_{k-1}, v_{k}\right) / u, v, v_{1}, \ldots v_{k-1}, v \in V\right\}$, $I_{k(e)}^{k}\left(v_{i}, v_{j}\right)$ is defined as $\sup \left\{I_{K(e)}\left(u, v_{1}\right) \wedge I_{K(e)}\left(v_{1}, v_{2}\right) \wedge I_{K(e)}\left(v_{2}, v_{3}\right) \ldots, I_{K(e)}\left(v_{k-1}, v_{k}\right) / u, v, v_{1}, \ldots v_{k-1}, v \in V\right\}$ and $F_{K(e)}^{k}\left(v_{i}, v_{j}\right)$ is defined as $\inf \left\{F_{K(e)}\left(u, v_{1}\right) \vee F_{K(e)}\left(v_{1}, v_{2}\right) \vee F_{K(e)}\left(v_{2}, v_{3}\right) \ldots, F_{K(e)}\left(v_{k-1}, v_{k}\right) / u, v, v_{1}, \ldots v_{k-1}, v \in V\right\}$.

Definition 2.15. Two vertices that are joined by a path is called connected (SVNG) graph.

Definition 2.16. Let $u$ be a vertex in an neutrosophic soft graph $G=(V, E)$, then $N(u)=\{v: v \in V\}$ and $(u, v)$ is a strong arc is called neighborhood of $u$.

Definition 2.17. A vertex $u \in V$ of an neutrosophic soft graph $G=(V, E)$ is said to be an isolated vertex if $T_{K(e)}(u, v)=0$ and $I_{K(e)}(u, v)$ and $F_{K(e)}(u, v)=0$, thus an isolated vertex does not dominated any other vertex in $G$.

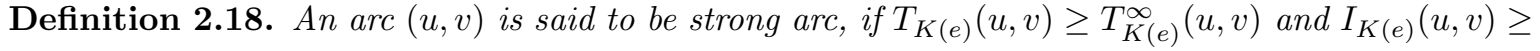
$I_{K(e)}^{\infty}(u, v)$ and $F_{K(e)}(u, v) \geq F_{K(e)}^{\infty}(u, v)$.

\section{Domination of Single Valued Neutrosophic Graph}

Definition 3.1. Let $G=(V, E)$ be an $(S V N G)$ on $V$. Let $u, v \in V$, we say that $u$ dominates $v$ in $G$ if there exists an strong arc between them.

Note 1:

1) For any $u, v \in V$, if $u$ dominates $v$ then $v$ dominates $u$ and hence domination is a symmetric relation on $V$.

2) For any $v \in V, N(v)$ is precisely the set of all vertices in $V$ which are dominated by $v$.

3) If $T_{K(e)}(u, v)<T_{K(e)}^{\infty}(u, v)$ and $I_{K(e)}(u, v)<I_{K(e)}^{\infty}(u, v)$ and $F_{K(e)}(u, v)<F_{K(e)}^{\infty}(u, v)$, for all $u, v \in V$ and then the only dominating set of $G$ is $V$.

Definition 3.2. A subset $S$ of $V$ is called a dominating set in $G$ if for every vertex $v \in V-S$ there exists $u \in S$ such that $u$ dominates $v$ for all $u, v \in V$.

Definition 3.3. A dominating set $S$ of an $(S V N G)$ is said to be minimal domiating set if no proper subset of $S$ is a dominating set. for all $u, v \in V$.

Definition 3.4. Minimum cardinality among all minimal dominating set is called lower domination number of $G$, and is denoted by $\sum_{S \in V}\left(d_{N S}(G)\right) \forall u, v \in V$.

Maximum cardinality among all maximum dominating set is called upper domination number of $G$, and is denoted by $\sum_{S \in V}\left(D_{N S}(G)\right) \forall u, v \in V$.

Example 3.1. Consider an (SVNG) graph $G=(V, E)$, such that $V=\{a, b, c, d\}$ and $E=\{(a b),(b c),(c d),(d a),(a c)\}$. 


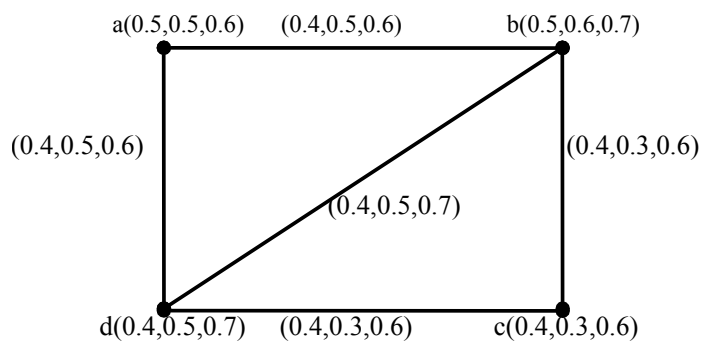

SINGLE VALUED NEUTROSOPHIC GRAPH

Figure 1

For the example (1) the dominating sets are $\{(a, b),(b, c),(c, d),(d, a),(a, b, c),(d, c, a),(b, d, a),(d), b\}$ the minimum dominating set $\{d\}$. The Maximum dominating set is $\{a, b\}$. The minimum dominating number is 0.6. The maximum dominating number 1.4 .

For Figure 1, domination number is $\sum_{S \in V}\left(d_{N S}(G)\right)=0.6$. and $\sum_{S \in V}\left(D_{N S}(G)\right)=1.4$.

Definition 3.5. Two vertices in an $(S V N G)$ graph, $G=(V, E)$ are said to be independent if there is no strong arc between them.

Definition 3.6. A subset $S$ of $V$ is said to be independent set of $G$ if $T_{K(e)}(u, v)<T_{K(e)}^{\infty}(u, v)$ and $I_{K(e)}(u, v)<I_{K(e)}^{\infty}(u, v)$ and $F_{K(e)}(u, v)<F_{K(e)}^{\infty}(u, v) \forall u, v \in S$.

Definition 3.7. An indepentent set $S$ of $G$ in an neutrosophic soft graph is said to be maximal independent, if for every vertex $v \in V-S$, the set $S \cup\{v\}$ is not independent.

Definition 3.8. The minimum cardinality among all maximal independent set is called lower independence number of $G$, and it is denoted by $\sum_{S \in V}\left(i_{N S}(G)\right)$. The maximum cardinality among all maximal independent set is called lower independence number of $G$, and it is denoted by $\sum_{S \in V}\left(I_{N S}(G)\right)$.

Example 3.2. Consider an above example for an $(S V N G)$ graph $G=(V, E)$, such that $V=\{a, b, c, d\}$ and $E=\{(a, b),(b c),(c d),(d a),(a c)\}$.

For the Example (2) minimum independent dominating set is $\{a, c\}$ and (2) maximum independent dominating set is $\{a, c\}$. Then the $\sum_{S \in V}\left(i_{N S}(G)\right)=1.55$ and $\sum_{S \in V}\left(I_{N S}(G)\right)=1.55$

Theorem 3.9. A dominating set $S$ of an $S V N G, G=(V, E)$ is a minimal dominating set if and only if for each $d \in S$ one of the following conditions holds.

(i) $d$ is not a strong neighbor of any vertex in $S$.

(ii) There is a vertex $v \in V-\{S\}$ such that $N(u) \cap S=d$.

Proof. Assume that $S$ is a minimal dominating set of $G=(V, E)$. Then for every vertex $d \in S$, $S-\{d\}$ is not a dominating set and hence there exists $v \in V-(S-\{d\})$ which is not dominated by any vertex in $S-\{d\}$. If $v=d$, we get, $v$ is not a strong neighbor of any vertex in $S$. If $v \neq d, v$ is not dominated by $S-\{v\}$, but is dominated by $D$, then the vertex $v$ is strong neighbor only to $d$ in $S$. That is, $N(v) \cap S=d$. Conversely, assume that $S$ is a dominating set and for each vertex $d \in S$, one of the two conditions holds, suppose $S$ is not a minimal dominating set, then there exists a vertex $d \in S, S-\{d\}$ is a dominating set. Hence $d$ is a strong neighbor to at least one vertex in $S-\{d\}$, the condition one does not hold. If $S-\{d\}$ is a dominating set then every vertex in $V-S$ is a strong neighbor at least one vertex in $S-\{d\}$, the second condition does not hold which contradicts our assumption that at least one of thse conditions holds. So $D$ is a minimal dominating set.

Theorem 3.10. Let $G$ be an $S V N G$ without isolated vertices and $S$ is a minimal dominating set. Then $V-S$ is a dominating set of $G=(V, E)$. 
Proof. $S$ be a minimal dominating set. Let $v$ be a any vertex of $S$. Since $G=(J, K$,$) has no isolated$ vertices, there is a vertex $d \in N(v)$. v must be dominated by at least one vertex in $S-v$, that is $S-v$ is a dominating set. By above theorem, it follows that $d \in V-S$. Thus every vertex in $S$ is dominated by at least one vertex in $V-S$, and $V-S$ is a dominating set.

Theorem 3.11. An independent set is a maximal independent set of $S V N G, G=(V, E)$ if and only if it is independent and dominating set.

Proof. Let $S$ be a maximal independent set in an SVNG, and hence for every vertex $v \in V-S$, the set $S \cup v$ is not in dependent. For every vertex $v \in V-S$, there is a vertex $u \in S$ such that $u$ is a strong neighbor to $v$. Thus $S$ is a dominating set. Hence $S$ is both dominating and independent set.

Conversely, assume $S$ is both independent and dominating. Suppose $S$ is not maximal independent, then there exists a vertex $v \in V-S$, the set $S \cup v$ is independent. If $S \cup v$ is independent then no vertex in $S$ is strong neighbor to $v$. Hence $S$ cannot be a dominating set, which is contradiction, Hence $S$ is a maximal independent set.

Theorem 3.12. Every maximal independent set in an $S V N G, G=(V, E)$ is a minimal dominating set.

Proof. Let $S$ be a maximal independent set in a NSG, by previous theorem, $S$ is a dominating set. Suppose $S$ is not a minimal dominating set, then there exists at least one vertex $v \in S$ for which $S-v$ is a dominating set, But if $S-v$ dominates $V-S-(v)$, then at least one vertex in $S-v$ must be strong neighbor to $v$. This contradicts the fact that $S$ is an independent st of $G$. Therefore, must be a minimal dominating set.

\section{PRODUCT DOMINATION IN SINGLE VALUED NEUTROSOPHIC GRAPH}

Definition 4.1. Let $G=(V, E)$ be an $S V N G$, If

(i) $T_{K}(x y) \leq\left\{T_{J}(x) \times T_{J}(y)\right\}$,

(ii) $I_{K}(x y) \leq\left\{I_{J}(x), I_{J}(y)\right\}$,

(iii) $F_{K}(x y) \leq\left\{F_{J}(x), F_{J}(y)\right\}$

and $0 \leq T_{K}(x y)+I_{K}(x y)+F_{K}(x y) \leq 3 . \forall u v \in V$. where $\times$ represent ordinary multiplication, the the $S V N G G$ is called the product $S V N G$.

Remark 4.2. If $G=(V, E)$ is an $S V N G$, because $T_{J}, F_{J}, I_{J}$ are less then or equal to 1 , it follows that,

(i) $T_{K}(x y) \leq\left\{T_{J}(x) \times T_{J}(y)\right\} \min \left\{T_{J}(x), T_{J}(y)\right\}$

(ii) $I_{K}(x y) \leq\left\{I_{J}(x), I_{J}(y)\right\} \min \left\{I_{J}(x), I_{J}(y)\right\}$

(iii) $F_{K}(x y) \leq\left\{F_{J}(x), F_{J}(y)\right\} \max \left\{F_{J}(x), F_{J}(y)\right\}$ for all $x, y \in V$.

Thus, every product of single valued neutrosophic graph is an single valued neutrosophic graph.

Example 4.1. Let $G=(V, E)$ be a product $S V N G$. Let the set be $V=\{a, b, c, d\}$ and $T_{J}(a)=$ $\{0.5,0.6,0.7\}, T_{J}(b)=\{0.6,0.7,0.4\}, T_{J}(c)=\{0.4,0.3,0.2\}, T_{J}(d)=\{0.5,0.4,0.6\}$ This example showing on figure 2 


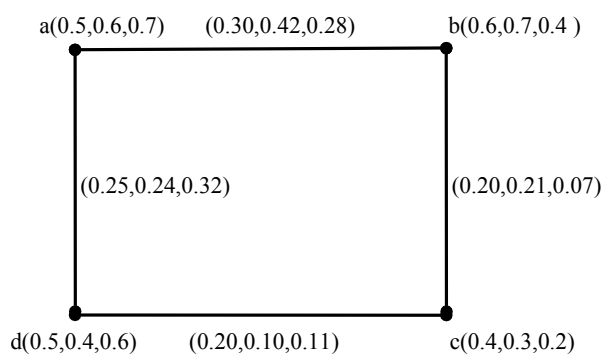

PSVNG

Figure 2

Definition 4.3. A product $S V N G G=(V, E)$ is said to be complete if

(i) $T_{K}(x y)=\left\{T_{J}(x) \times T_{J}(y)\right\}$

(ii) $I_{K}(x y)=\left\{I_{J}(x), I_{J}(y)\right\}$

(iii) $F_{K}(x y)=\left\{F_{J}(x), F_{J}(y)\right\}$ for all $x, y \in V$

Definition 4.4. The complement of $P S V N G G=(V, E)$ is an $P S V N G \bar{G}=(\bar{V}, \bar{E})$ is an $S V N G$ where,

(i) $T_{J}=\bar{T}_{J}, T_{K}(x y)=\left\{T_{J}(x) \times T_{J}(y)-T_{K}(x y),\right\}$

(ii) $I_{J}=\bar{I}_{J}, I_{K}(x y)=\left\{I_{J}(x) \times I_{J}(y)-I_{K}(x y),\right\}$

(iii) $F_{J}=\bar{F}_{J}, F_{K}(x y)=\left\{F_{J}(x) \times F_{J}(y)-F_{K}(x y)\right.$. $\}$ for all $x, y \in E$.

Definition 4.5. An arc $(x, y)$ is said to be strong arc in PSVNG if $T_{K}(x y)=\left\{T_{J}(x) \times T_{J}(y)\right\}$, $I_{K}(x y)=\left\{I_{J}(x), I_{J}(y)\right\}$ and $F_{K}(x y)=\left\{F_{J}(x), F_{J}(y)\right\}$ for all $x, y \in V$

Definition 4.6. Let $X$ be a vertex in $P S V N G G=(V, E)$ then $N(u)=\{v: v \in V:(u, v)$ isastrongarc $\}$ is called neighborhood of $u . x, y \in V$ In Particular case, if $T_{K}(x, y)=0, I_{K}(x, y)=0, F_{K}(x, y)=0$ for all $u \in V$.

Definition 4.7. A vertex $x \in V$ of an $P S V N G G=(V, E)$ is said to be an isolated vertex if $T_{K}=$ $0, F_{K}=0, I_{K}=0$ for all $x, y \in V$.

Definition 4.8. An arc $(x, y)$ is said to be a strong arc, if $T_{K}(x, y) \gtrless T_{K}^{\infty}(x y), I_{K}(x, y) \gtrless I_{K}^{\infty}(x y)$, and $F_{K}(x, y) \gtrless F_{K}^{\infty}(x y)$ for all $x, y \in V$.

Definition 4.9. Let $G=(V, E)$ be an $P S V N G$ on $V$. Let $x, y \in V$. we say that $x$ dominates $y$ in $G$ if there exists a strong arc between them for all $x, y \in V$.

Remark 4.10. (1) for any $x, y \in V$, if $x$ is dominates $y$ then $y$ dominates $x$ hence domination is a symmetric relation on $V$.

(2) for any $x \in V, N(x)$ is precisely the set of all vertices in which are dominate by $v$.

(3) if $T_{K}(x, y)<T_{K}^{\infty}(x y), I_{K}(x, y)<I_{K}^{\infty}(x y), a n d F_{K}(x, y)<F_{K}^{\infty}(x y)$ for all $x, y \in V$. then the only dominating set of Gis $V$.

Definition 4.11. A subset $S$ of $V$ is called a dominating set in PSVNG $G$ if for every vertex $v \in V-S$ there exists $u \in S$ such that $u$ dominates $v$ for all $x, y \in V$.

Definition 4.12. A dominating set $S$ of an PSVNG is said to be minial dominating set if no proper subset of $S$ is a dominating set for all $x, y \in V$.

Minimum cardinality among all minimal dominating set is called lower domination number of $G$, and is denoted by $\sum_{S \in V} d_{P S V N}(G)$.

Maximum cardinality among all maximal dominating set is called upper domination number of $G$, and is denoted by $\sum_{S \in V} D_{P S V N}(G)$. 
Example 4.2. Consider a $P S V N G G=(V, E)$ for figure(3), such that $V=\{a, b, c, d, e\}$ and $E=$ $\{(a b),(b c),(c d),(d e),(a c),(b d)\}$

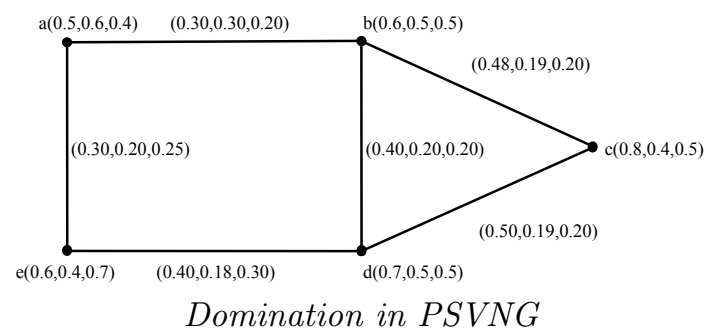

Figure 3

Here $(c d)$ weak arc, then the dominating sets are $S_{1}=\{a, b\}, S_{2}=\{a, b, c\}, S_{3}=\{d, b\} S_{4}=$ $\{c, d, a\} S_{5}=\{c, e\} S_{6}=\{a, b, d\} S_{7}=\{d, c, e\}, S_{8}=\{a, b, e\}$ then vertex cardinality of all dominating sets,

$S_{1}=1.65, S_{2}=1.70, S_{3}=1.65, S_{4}=2.55, S_{5}=1.50, S_{6}=2.50, S_{7}=2.35, S_{8}=2.30$,

Here. $S_{5}$ is a minimum dominating set of minimum cardinality and $d_{P S V N G}(G)=1.50, S_{2}$ is a minimal dominating set of maximum cardinality and $D_{P S V N G}(G)=1.7$

Definition 4.13. Let $G=(V, E)$ be an PSVNG without isolated vertices, $A$ set $S$ is a total dominating set if for every vertex $u \in V$, then there exists a vertexx $v \in S, u \neq v$ such that $u$ dominates $v$ for all $x, y \in V$.

The minimum cardinality among all minimum total dominating set is called lower total domination number of $G=(V, E)$ and is denoted by $t_{P B}(G)=\sum_{s \in V} S(G)$.

The maximum cardinality among all maximum total dominating set is called lower total domination number of $G=(V, E)$ and is denoted by $T_{P B}(G)=\sum_{s \in V} S(G)$.

The proof of the following results are similar to that of the above, hence omitted.

Theorem 4.14. A dominating set $D$ of an PSVNG, $G=(V, E)$ is a minimal dominating set if and only if for each $v \in S$ one of the following conditions are satisfied. (i) $v$ is not a strong neighbor of any vertex in $S$.

(ii) There exists a vertex $v \in V-\{S\}$ such that $N(u) \cap D=\{v\}$.

Theorem 4.15. Let $G$ be an PSVNG without isolated vertices and $S$ is a minimal dominating set. Then $V-S$ is a dominating set of $G=(V, E)$.

Theorem 4.16. An independent set is a maximal independent set of $P S V N G, G=(V, E)$ if and only if it is independent and dominating set.

Theorem 4.17. Every maximal independent set in an $P S V N G, G=(V, E)$ is a minimal dominating set.

\section{TRIPLE CONNECTED DOMINATION NUMBER OF A SVNG}

Definition 5.1. Let $G=(V, E)$ be a $S V N G$ any three vertex lie on a path is called a triple connected $S V N G$.

Definition 5.2. A subset $S$ of $V$ of a non-trival graph of $G=(V, E)$ is said to be an triple connected domination set, if $S$ is a dominating set and the induced subgraph $\langle S\rangle$ is triple connnected. minimum cardinality among all minimal dominating set is called lower domination number of $G$, and is denoted by $T_{S V N}(G)=\sum_{u \in V} S(G)$. 
Example 5.1. Consider a $S V N G$ for a figure (4).

Let $V=\{a, b, c, d, e\}$ and $E=\{(a b),(b c),(c d),(d e),(e f),(a f),(b d),(d f)\}$. for the figure $(c d)$ is weak arc.

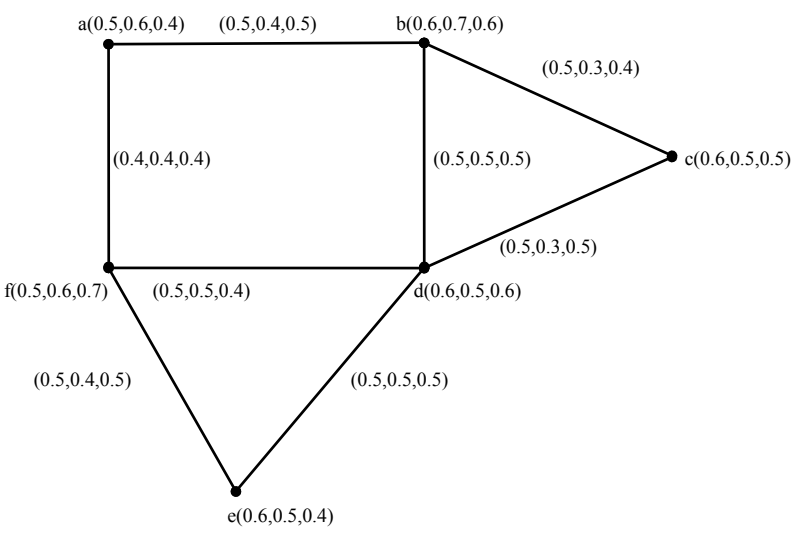

Triple Connected Domination in SVNG

Figure 3

Here the triple connected dominating sets are

$S_{1}=\{a, b, c\}, S_{2}=\{a, b, d\}, S_{3}=\{d, b, e\}, S_{4}=\{d, b, c\}, S_{5}=\{e, d, c\}, S_{6}=\{a, b, e\}$

Here, $S_{1}$ and $S_{6}$ are minimum dominating sets of minimum domination number $T_{S V N}=2.3$

Remark 5.3. Single valued neutrosophic triple connected dominating set does not exists for all graphs and if exists the $T_{S V N}(G) \geqq 3$ (Since all arcs arc strong arcs)

Remark 5.4. The complement of the SVNG triple connected dominating set need not be a triple connected dominating set.

Remark 5.5. Every SVNG triple connected dominating set is dominating set, but not conversely,

\section{Conclusion}

In this work, we derived the domination number of single valued neutrosophic graphs and elaborate them with suitable examples by using strength of path and strength of connectedness. Further, we investigate some remarkable properties of product single valued neutrosophic domination number and triple connected domination graph and the proposed concepts are described with suitable examples.

\section{REFERENCES}

[1] R. B. Allan and R. Laskar, On domination and independent domination numbers of a graph, Discrete Mathematics, Vol. 23, No. 2 (1978), 73-76.

[2] K. R. Bhutani, A. Rosenfeld, Strong arcs in fuzzy graphs, Information Sciences 152 (2003),319 - 322.

[3] A. Nagoorgani, and R. Jahir Hussain, Fuzzy independent dominating set, Advances in fuzzy sets and Systems 2(1) (2007), 99-108.

[4] A. Somasundram and S. Somasundram, Domination in Fuzzy Graphs - I , pattern Recognition Letters, 19(1998), $787-791$.

[5] S. Mohinta, T.K.Samantv, An introduction to fuzzy soft graphs, Mathematica Moravica 19-1(2015).

[6] M. Akram, S. Nawaz, On fuzzy soft graphs, Italian Journal of Pure and Applied Mathematics 34 (2015) 497-514.

[7] M. Akram, Sundas Shahzadi, Neutrosophic soft graphs with application, Journal of Intelligent \& Fuzzy Systems, vol. 32 , no. 1 , pp. $841-858,2017$

[8] M. Akram, Gulfam Shahzadi, Operations on Single-Valued Neutrosophic Graphs,Journal of uncertain systems, Vol.11, No.1, pp. 1-26, 2017.

[9] M. Akram, Neutrosophic competition graphs with applications,Journal of Intelligent and Fuzzy Systems, Vol. 33, No. 2, pp. 921-935, 2017.

[10] M. Akram, Shahzadi. S, Representation of Graphs using Intuitionistic Neutrosophic Soft Sets,Journal of Mathematical Analysis, Vol 7, No 6 (2016), pp 31-53. 
[11] Akram, M.; Malik, H.M.; Shahzadi, S.; Smarandache, F. Neutrosophic Soft Rough Graphs with Application. Axioms 7, 14 (2018).

[12] Ali, M.I., Feng, F., Liu, X.Y., Min W.K., and Shabir, M. (2009) On some new operations in soft set theory, Computers and Mathematics with Applications, 57, 1547-1553.

[13] Broumi, S., Delib, I., and Smarandachec, F. Neutrosophic refined relations and their properties, Neutrosophic refined relations and their properties Neutrosophic Theory and Its Applications.,(2014), pp 228-248.

[14] Deli, I., and Broumi, S. Neutrosophic soft relations and some properties, Annals of Fuzzy Mathematics and Informatics (AFMI), (2014), pp 1-14.

[15] Dhavaseelan, R., Vikramaprasad, R., and Krishnaraj, V. Certain types of neutrosophic graphs. Int Jr. of Mathematical Sciences and Applications, 5(2)(2015), pp 333-339.

[16] Maji, P. K. (2013). Neutrosophic soft set. Annals of Fuzzy Mathematics and Informatics, 5(1), 157-168.

[17] F. Smarandache, Neutrosophic set, a generalisation of the intuitionistic fuzzy sets, Inter. J.Pure Appl. Math. 24 (2005) 287-297.

[18] F. Smarandache, Neutrosophic Graphs, in his book Symbolic Neutrosophic Theory, Europa, Nova.

[19] D.A Molodtsov, Soft set theory-first results, Computers and Mathematics with Application 37 (1999), pp 19-31.

[20] D.A. Moltdtso, The Theory of Soft Sets(in Russain), URSS Publishers, Moscow, 2004.

[21] Rosenfeld. A Fuzzy Graphs. In Fuzzy Sets and their Applications to Cognitive and Decision Processes. Zadeh LA,FuKS, Shimura M, editors. New York Academic press, 1975. pp 77-95.

[22] P.K. Maji, A.R. Roy, R. Biwas, Fuzzy soft sets, The Journal of Fuzzy Mathematics 9(3)(2001).

[23] P.K. Maji, A.R. Roy, R. Biwas, An application of soft sets a decision making problem, Computer and Mathematics with Application 44(8-9) (2002) 1077-1083.

[24] Said, Broumi, and Smarandache, F. (2013). Intuitionistic neutrosophic soft set. Journal of Information and Computer Science, 8(2), 130-140.

[25] Said Broumi, Smarandache, F., Talea, M., and Bakali, Single Valued Neutrosophic Graphs: Degree,Order and Size, 2016 IEEE International Conference on Fuzzy Systems (FUZZ-IEEE).

[26] Said Broumi, Mohamed Talea, Assia Bakali, Florentin Smarandache, Single Valued Neutrosophic Graphs. The Journal of New Theory, 2016(10),861-101

[27] Said Broumi, Florentin Smarandache, Mohamed Talea, Assia Bakali, An Introduction to Bipolar Single Valued Neutrosophic Graph Theory. Applied Mechanics and Materials, 2016(841)184-191.

[28] Said Broumi, Florentin Smarandache, Mohamed Talea, Assia Bakali, An Isolated Bipolar Single-Valued Neutrosophic Graphs.Information Systems Design and Intelligent Applications, 2018(672)816-822.

[29] Wang, H., Smarandache, F., Zhang, Y., and Sunderraman, R. (2010). Single valued neutrosophic sets. Multispace and Multistructure Vol 4, pp 410-413.

[30] Zadeh, L. A. (1965). Fuzzy sets. Information and Control, Vol 8 No 3, 338-353. 